\title{
以临床案例为导向的检验班分析化学教学实践
}

\author{
钮因尧，陈聪颖 ${ }^{*}$ \\ 上海交通大学医学院基础医学院, 上海 200025
}

\begin{abstract}
摘要: 将分析化学内容与医学实际紧密结合, 开展以临床案例为导向的教学, 有利于激发学生的学习兴趣, 加深对 概念的理解, 提高分析、归纳、综合的能力, 及在此基础上, 增强应用专业知识解决医学实际问题的能力; 同时, 使学生增强责任感、接受辩证的思想、树立自主学习的理念和逐渐养成终生学习的习惯。
\end{abstract}

关键词: 分析化学; 临床案例; 自主学习

中图分类号: G64; O6

\section{Practice of Analytical Chemistry Teaching Guided by Clinical Cases for Medical Laboratory Major Students}

\author{
Yinyao Niu, Congying Chen * \\ College of Basic Medical Sciences, School of Medicine, Shanghai Jiao Tong University, Shanghai 200025, China.
}

\begin{abstract}
The clinical case-guided teaching, to combine analytical chemistry contents with medical practice, could excite students' interest in learning, deepen concept understanding and improve the abilities of analysis, induction and synthesis, and then of applying professional knowledge to solve medical practical problems. Meanwhile, it helps students enhance their sense of responsibility, accept the idea of dialectics and the concept of self-access learning, and gradually develop the habit of lifelong learning.
\end{abstract}

Key Words: Analytical chemistry; Clinical case; Self-access learning

\section{1 前言}

分析化学是许多理工医科院校相关专业的常设课程, 课程的教学任务是介绍现代各种分析方法 的基本原理及其应用。然而, 医学院的检验专业对此课程的教学要求有其自身的专业特点。临床医 学检验的重要任务是为疾病诊断、鉴别诊断、疾病治疗和疗效观察提供可靠的依据。作为医学检验 专业的必修专业基础课, 分析化学传授的检测方法在实际中的应用知识, 必须与临床医学诊断、治 疗、预防等密切相关。若课程教学中的应用案例缺乏医学特色, 很难使学生体会到这些专业知识的 临床应用价值, 也无法引起学生对该课程的学习兴趣。在当今第三次教育革命的浪潮中, 授课教师 转变教学理念, 以学生为主体, 坚持目标引导, 积极开展教学探索, 通过多种教学方式 ${ }^{[1-3]}$ 激发学生 的学习积极性, 则能使学生把被动学习转变为主动求知 ${ }^{[4]}$ 。在化学及药学等非化学类专业中实施的 分析化学案例教学法, 充分体现专业特色以及与生活实践、学科前沿的紧密联系, 拉近 “学” 与用” 的距离, 活跃课堂气氛, 并在教师教学和学生学习之间建立良好的互动关系, 改善了教学效果 ${ }^{[5-7]}$ 。

收稿: 2020-07-31; 录用: 2020-08-13; 网络发表: 2020-09-14

“通讯作者, Email: chency@sjtu.edu.cn

基金资助：上海交通大学教学发展中心教学发展基金项目(CTLD19J 0002) 
为此, 我们在医学检验班的分析化学课程中, 将分析化学与医学紧密结合, 开展以临床案例为导向 的教学实践, 获得了学生的认可, 现针对以下四个方面作一些具体阐述。

\section{2 分析化学课程与临床案例相结合的教学探索}

作为检验班分析化学教学导向的临床案例, 来源于上海交通大学医学院附属医院急诊科的一手 病例, 经授课教师改编而成, 具有真实性和医学专业性, 而其中设置的问题则体现了较强的分析化 学专业性。

\section{1 融入案例, 激发学生的学习兴趣, 增强责任感}

分析化学课程的教学主要涉及各种分析技术的方法和原理, 内容相对比较枯燥, 学生的主动学 习意愿不强。因此, 我们在第 1 节绪论课中, 就引入临床真实案例, 譬如 “咳嗽咯血被误诊导致不 幸”, 以期提高学生的学习兴趣。该案例中患者因咳嗽咯血去当地医院就诊时, 未做任何检查, 直 接按感冒咳嗽病例处理, 几天后病情加重急诊抢救。随后案例展示检验报告, 显示多项指标异常, 尤其血钾含量极高, 最终病人因肾功能衰竭导致不幸。通过该案例, 告诉学生: 1) “临床化学” 分 析检验报告的意义(为诊断病情、早做对症治疗以治愈或延缓病情提供关键依据)；2）如何得到有效 可靠的分析数据(应用多种化学分析技术的原理和测试方法, 如电化学、色谱分析原理和检测手段); 3) 如何评价分析数据的可靠性(涉及分析化学中误差与分析数据处理方法等)。通过对案例的分析、 引导, 使医学检验专业的学生体会到分析化学与检验专业是密切相关的, 并进一步意识到分析化学 中的原理及其临床应用知识, 对救治患者生命以及在其未来职业生涯中的重要性, 增强了学生的责 任感, 加强了课程的思政建设。

\section{2 利用案例加深学生对 “量” 概念的理解, 接受辩证的思想}

分析化学是 “从事科学研究的科学”, 临床生化指标和毒物的检测、药物治疗时的血药浓度监 测等, 是分析化学作为一种工具在医学中的具体应用, 其中大部分目标物的分析都涉及定量, 而 “量” 的概念在医学检验中更凸显出它的相对性。

血 $\mathrm{pH}$ 、血糖、血尿酸等重要生化指标是临床诊断的重要依据之一, 这些生化指标都有一个临床 正常范围。人们的检测指标, 从数值本身来讲是绝对的; 若相对此 “正常范围” 偏高或偏低, 则提 示人体健康可能出现问题。生活中人们习惯于对物质有 “无毒” 与 “有毒” 的绝对之分, 但是从医 学的角度思考, 自然界中并不存在对人体绝对 “无毒” 或绝对 “有毒” 的物质。譬如, 食盐是人类 不可缺少的 “无毒” 物质, 但如果一次摄入 15-60 g 会损害机体, 一次摄入 200-250 g, 即可因电解 质严重紊乱而导致死亡。反之, “剧毒” 物质, 如砒霜等, 在低于中毒剂量时, 也可作为临床药物 使用。而很多药物, 如秋水仙碱、阿托品、可待因等, 在过量使用时, 也会引起中毒。为了强调过 量服药的危害性, 加深理解 “量” 的概念, 体会 “相对性” , 我们在教学中引入有关药物性损伤的 案例和说明: 患者 3 日前因 “落枕” 自服 50 片止痛片(主要成分对乙酰氨基酚 $650 \mathrm{mg} /$ 片)后出现乏 力嗜睡, 伴恶心呕吐至医院急诊, 完善相关检查后, 诊断为急性药物性肝衰竭及电解质代谢紊乱。 对乙酰氨基酚具有解热镇痛作用, 是常用感冒药和止痛片(如泰诺、日夜百服宁等)的有效成分之一。 通过药品说明书可以了解每片(或粒)药中对乙酰氨基酚的质量, 并进一步获得在过量服用某种感冒药 或者混合使用多种感冒药的情况下, 可能出现中毒的提示。随后简要说明过量服用止痛片导致药物性 肝损伤引起肝衰竭, 并启发学生在今后的检验工作中要树立 “量” 的相对性概念。进一步引导学生 讨论: 1) 若自行用药, 服药前是否需要了解药物的主要成分以及含量(有些病患服药时并不关心药 物的成分和含量, 也缺乏 “量” 的概念, 误认为服药量越大, 治疗效果越好, 因服药过量引起的中 毒事件时有发生), 并得出结论: 人们在服用前有必要了解药物, 亟需提升医学健康素质; 2) 若止痛 片与其他药物同时使用, 可能会发生什么情况, 引出影响体内药物含量的因素(譬如, 药物相互作用 影响药物的吸收代谢, 可能导致血药浓度改变; 或各种药物中含有相同化学成分导致临床反应过强), 而这些影响因素在课程后期设计临床样本中化学成分的分析方案时可能需要加以考虑。通过案例, 
让我们深刻体会到 “量” 的相对性, 学会辩证地看待问题, 同时也要注意, 对于 “量” 的相对性也 不能片面夸大，教师在授课时要具体问题具体分析，把握好尺度。

\section{3 开展案例讨论, 训练学生的分析、归纳、综合能力, 使其养成终生学习的习惯}

除传统课堂授课外, 我们还结合课程知识安排了一定教学时数的临床案例讨论课, 在课堂讨论 两周前将案例发给学生。由于案例涉及分析化学中多个教学知识点和实际应用, 学生需组队分工合 作, 针对问题查阅资料, 分析归纳总结, 在课堂展示时, 组间提问, 最后完成书面报告。譬如, 案 例 “糖尿病人的困惑”, 在简述患者病因、症状以及治疗方案后, 提供辅助检查数据(血气分析、电 解质、血糖、尿常规、肾功能等)。案例中与分析化学课程关系密切的内容是各类临床检查数据的测 定方法, 因此, 我们设计了多个问题供学生组织讨论。譬如, 比较临床上两种葡萄糖常规检测法——葡 萄糖氧化酶法、己糖激酶法的优缺点。针对这个问题, 通过查阅文献, 学生首先掌握了对于单一葡 萄糖样品所采用的分析方法及其特点; 在此基础上, 结合临床样品(尿、血)的特点, 了解人体内葡萄 糖(尿糖、血糖)检测的方法及其特点, 进一步通过分析各种方法的适用性, 最后归纳、总结两种分析 检测方法的优缺点(譬如, 用葡萄糖氧化酶法检测尿毒症、痛风等标本时, 因尿酸等物质干扰氧化酶 反应, 导致结果偏低; 而己糖激酶法的检测结果基本不受这些临床因素的干扰)。通过课堂提问与组 间讨论, 学生能进一步认识到针对具体样品选择分析方法时, 还需要考虑各种实际影响因素, 对临 床检测葡萄糖的分析方法有了更深入的认识。

随着实验技术和理论方法的不断发展、完善, 每一门学科的知识内容处在不断的更新中。在将 分析化学与临床实际紧密融合的同时, 使学生及时了解相关的科学研究前沿技术和知识, 可以充分 体会分析方法的发展, 培养学生养成终身学习的好习惯。在上述案例中, 我们还设问: 血气分析、 血糖等常规检测的发展趋势是什么? 有哪些最新应用成果? 针对该问题, 部分小组分别介绍和探讨了 血糖、尿糖监测仪的基本检测原理、目前常规医院用和家用监测仪的共同点与区别、血糖仪的发展 趋势(无创检测) 以及血糖研究的前沿(智能调控), 以及血气分析、酮体和尿酸的检测方法与原理、目 前临床常规使用的全自动生化分析仪和即时检测血酮体仪、尿酸生物传感器等检测仪器。学生在讨 论中不仅巩固了相关的检测原理等理论知识, 而且深刻体会到分析化学在医学检验中的重要意义, 促进了探究式学习的开展, 体现教学的高阶性。

我们将该案例用于 2018 和 2019 级医学检验班的分析化学讨论课。整体上, 学生围绕案例中的 讨论题精心准备, 课堂展示时基本能抓住问题要点, 在回答提问时思维敏捷并充分体现团队合作精 神; 同时对其他小组积极提问, 且提出的问题具有一定的深度, 课堂讨论气氛活跃, 从中看出学生 很享受积极互动的课堂氛围。案例(包括其中设置的题目)是否与课程基础知识紧密相关和案例中的 题目难度, 是影响课堂讨论效率的两个关键因素。为了评估讨论课的教学效果, 以便积累经验, 提 高教学质量, 我们从上述两方面, 对 140 名学生作了初步的问卷调查, 收到反馈 132 份, 结果见图 1、图 2。图 1 中认为案例较适合、适合、很适合用课程基础知识进行讨论的分别占 $33.33 \%$ 、41.67\%、

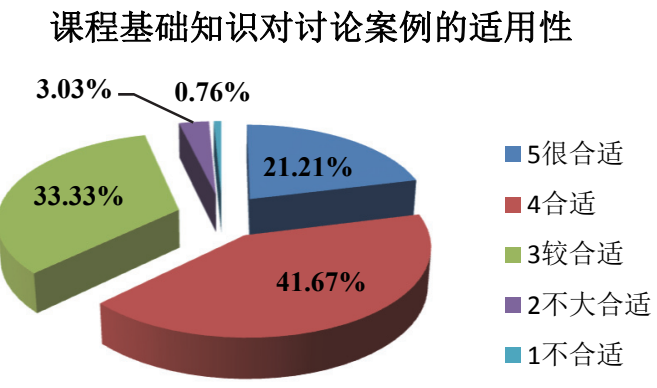

图 1 问卷调查结果 1

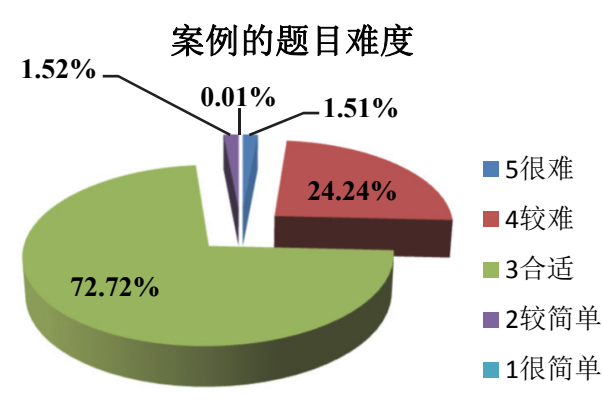

图 2 问卷调查结果 2 
$21.21 \%$, 三者之和超过 $96 \%$; 图 2 中认为案例中题目难度合适的占 $72.72 \%$ 、较难的占 $24.24 \%$, 说 明学生对讨论课的整体满意度较高。此外, 许多学生反映案例讨论课不仅使他们加深了对课程基础 知识点的理解、了解了本学科的发展前沿, 而且对分析、归纳、综合能力的提高具有明显帮助。

\section{4 案例引导文献研究, 提高学生应用知识解决实际问题的能力, 树立自主学习的理念}

案例教学法使学生置身于充满问题的真实情景中, 激励他们运用课程知识建立解决实际问题的 方法, 实现学以致用的教学目标。在医学检验专业的分析化学教学大纲中, 高效液相色谱法(HPLC) 的教学要求是学生在掌握 HPLC 基本原理、应用的基础上, 具有初步设计实际分析方案的能力。由 于分析化学中涉及仪器分析的内容比较抽象, 而且在教材和传统的常规教学中偏重于分析原理的介 绍, 学生对分析流程中的实际过程(包括为什么要对生物样本进行预处理和怎么处理、如何选择合理 的分离分析条件和合适的定量分析方法) 以及操作中的注意事项等感性认识不足, 因此, 在设计具体 分析方案时遇到很大的困难。为了解决这一难题, 我们尝试在课前向学生提供案例简介和具体的 HPLC 分析文献, 通过案例引导他们仔细阅读文献, 加深对整个分析过程的了解, 然后教师在课堂 上针对案例, 结合理论知识, 边讲解实验设计过程, 边通过提问引导学生思考各个步骤的目的或原 因, 同时进行集体讨论, 增强教学内容的实用性, 为培养学生应用专业知识解决实际问题的能力打 下基础。

百草枯的主要成分是一种联吡啶类化合物(1,1'一二甲基-4,4'一二氯二吡啶), 百草枯属高毒类除草 剂。患者就诊时百草枯的血浆浓度与中毒时间是评估患者预后的重要指标。通过测定临床血浆样本 并计算百草枯中毒严重指数, 可为临床治疗并预测预后提供科学依据。学生通过课前了解案例 “百 草枯中毒案” 并熟读文献 “反相高效液相色谱法测定人血浆百草枯浓度及其临床应用” ${ }^{[8]}$, 在听课过 程中至少收获以下几点: 1) 加深对血浆等临床生物样本分析的系统认识, 包括生物样本预处理的意 义、方法与特点、生物样本分析方法的建立过程和方法验证的内容与要求; 2) 深刻理解合适的 HPLC 分离分析条件和定量方法的选择。譬如, 根据待测物的化学结构以及反相色谱法的特点, 即固定相 极性较小, 适用于分离非极性或弱极性化合物, 故选择 $\mathrm{C}_{18}$ 反相色谱柱, 巩固了正相和反相 HPLC 分 离特点的区别。此外, 启发学生思考流动相中添加试剂庚烷磺酸钠的作用, 以及用磷酸-磷酸二氢钾 缓冲对调节 $\mathrm{pH}$ 的目的等等; 3) 领会实际操作过程中每一步骤的目的以及注意事项, 譬如, 为了考 查血浆样品处理后放置稳定性, 需 $-40{ }^{\circ} \mathrm{C}$ 保存并反复冻融 3 次后才处理、测定等。

课后学生反映通过案例引导文献阅读和分析, 不仅掌握了分析原理, 而且熟悉了实际的分析测 定过程。这种教学方式锻炼了学生的实际应用能力, 既为后续药物或毒物色谱分析的实验方案设计 提供方法参考, 又使学生树立起自主学习的理念。

\section{3 结语}

在以临床案例为导向的检验班分析化学教学实践基础上, 我们将总结案例教学经验, 利用医学 院的独特优势, 积累更多的医学素材, 不断发掘、完善临床案例, 充实案例库 ${ }^{[9]}$ 。同时, 积极引导学 生独立思考, 并为学生提供综合学习的机会和有效思辨的空间。此外, 拟进一步拓展案例至人体的 营养健康和生活的多个方面, 将 “医学、健康、生活” 的理念与分析化学教学更紧密结合。在激发 学生的学习兴趣、加深对概念的理解、提高分析、归纳、总结和解决实际问题能力的同时, 促使学 生增强责任感、接受辩证的思想、树立自主学习的理念和养成终身学习的习惯。这种以与理论紧密 结合的实践为导向的教学模式, 可为高等院校中其他课程的相关教改探索提供参考。

\section{参 考 文 献}

[1] 陈聪颖, 何伟娜, 盛慧球. 基础医学教育, 2017, 19 (8), 582 .

[2] 李娜, 李美仙, 王申林, 刘小云, 白玉, 朱志伟. 大学化学, 2020, 35 (1), 28. 
[3] 王琳, 袁东, 马文, 韩桂美, 丁梦丽, 董菁. 大学化学, 2019, 34 (7), 11.

[4] 陈薪安, 董海艳, 丁茂超. 基础医学教育, 2020, 22 (3), 232.

[5] 何婧琳, 曹忠, 李丹, 张玲, 谭淑珍, 陈平, 李伟. 大学化学, 2016, 31 (8), 27.

[6] 杨冬芝, 汤道权, 江相兰, 林奇泗. 西北医学教育, 2012, 20 (5), 977.

[7] 康晓红, 程志明, 李智, 刘博, 段武彪. 化学教育, 2016, 37 (2), 47.

[8] 彭怡军, 董维冲, 李浩然, 李德强, 张志清. 中国临床药理学杂志, 2018, 34 (3), 323.

[9] 陈聪颖, 钮因尧, 盛慧球. 基础医学教育, 2018, 20 (9), 732. 